\title{
Pulmonary Hemodynamics and Six-Minute Walk Test Outcomes in Patients with Interstitial Lung Disease
}

\author{
Osamu Nishiyama, Ryo Yamazaki, Hiroyuki Sano, Takashi Iwanaga, Yuji Higashimoto, \\ Hiroaki Kume, and Yuji Tohda
}

Department of Respiratory Medicine and Allergology, Kindai University Faculty of Medicine, 377-2 Onohigashi, Osakasayama, Osaka 589-8511, Japan

Correspondence should be addressed to Osamu Nishiyama; nishiyama_o@yahoo.co.jp

Received 6 February 2016; Accepted 4 May 2016

Academic Editor: Edith J. Filion

Copyright (C) 2016 Osamu Nishiyama et al. This is an open access article distributed under the Creative Commons Attribution License, which permits unrestricted use, distribution, and reproduction in any medium, provided the original work is properly cited.

\begin{abstract}
Background. Six-minute walk test (6MWT) has 3 measurement outcomes, which are walk distance, desaturation, and symptom. The aim of this study was to examine whether routinely measured right-heart catheter (RHC) data correlate with 6MWT outcomes in patients with interstitial lung disease (ILD). Methods. Between June 2010 and December 2012, consecutive patients with ILD who underwent evaluation, including pulmonary function test, hemodynamic studies with right-heart catheter, and 6MWT as routine general practice, were recruited. Correlates of 3 outcomes of 6MWT were examined to reveal significant predictors. Results. Fortysix patients consisting of 20 with idiopathic pulmonary fibrosis, 14 with collagen vascular disease associated ILD, and 12 with other idiopathic interstitial pneumonia were recruited (mean \% predicted FVC: $76.7 \pm 17.1 \%$ ). Several physiological variables, including mean pulmonary artery pressure (PAP) and pulmonary vascular resistance (PVR), were correlated with each 6MWT outcome. Stepwise multivariate regression analyses showed that \% predicted FVC and \% predicted DLco were independent predictors of walk distance $\left(r^{2}=0.35, p=0.0002\right)$. For $\mathrm{SpO}_{2}$ at the end of 6MWT, \% predicted DLco and PVR were selected as independent predictors $\left(r^{2}=0.46, p<0.0001\right)$. For dyspnea at the end of $6 \mathrm{MWT}, \%$ predicted DLco was only one predictor $\left(r^{2}=0.18\right.$, $p=0.005)$. Conclusion. Mean PAP had little impact on 6MWT outcomes in ILD patients who were nonselectively recruited, although PVR was one of predictors of desaturation.
\end{abstract}

\section{Introduction}

The 6-min walk test (6MWT) is the most frequently used exercise capacity assessment for patients with chronic lung disease. Exercise tolerance assessed with 6MWT is submaximal capacity because most patients do not achieve maximal effort during the test [1]. The test itself requires an unobstructed walking path but no exercise equipment and little advanced training to administer. It evaluates the integrated global response of the pulmonary, cardiovascular, and neuromuscular systems. Therefore, it is considered difficult to provide specific information regarding which system contributes to exercise limitation, something a cardiopulmonary exercise test would provide $[2,3]$.

However, correlates of a 6-min walk distance have been studied in several diseases. In the field of lung disease, chronic obstructive pulmonary disease (COPD) has been earnestly investigated. Several factors which predict a reduced 6min walk distance in patients with COPD were previously found. Not only decreased forced expiratory flow in one second $\left(\mathrm{FEV}_{1}\right)$ [1], but also older age [4], female sex [5], and higher body mass index $[5,6]$ were reportedly associated with reduced walk distance. In patients with interstitial lung disease (ILD), however, significant predictors of 6MWT outcomes have not been sufficiently investigated.

When performing 6MWT, not only distance walked but also $\mathrm{O}_{2}$ saturation and dyspnea rating are usually recorded. In patients with idiopathic pulmonary fibrosis (IPF), for example, both walk distance and desaturation at $6 \mathrm{MWT}$ are significant prognostic markers [7-11]. Thus, evaluating 6MWT outcomes is important in ILD as well. Moreover, desaturation during $6 \mathrm{MWT}$ is more severe for IPF than those 
with $\mathrm{COPD}$, even though resting oxygenation between the two is equivalent [12]. This might be a consequence of the differing pathophysiology between restrictive and obstructive lung disease. Mechanisms of exercise intolerance are also different, so the correlates of 6MWT outcomes should be investigated separately in obstructive and restrictive lung disease.

Recently, a high prevalence of pulmonary hypertension was found in IPF, and evaluating pulmonary artery pressure has been becoming important [13-16]. Moreover, pulmonary hypertension in ILD is associated with exercise-induced desaturation [14]. Therefore, pulmonary hemodynamics in ILD might be involved in reduced exercise tolerance and be associated with 6MWT parameters, including walk distance, desaturation, and symptoms. However, pulmonary artery pressure is not usually measured in clinical practice unless presence of pulmonary hypertension is strongly suspected.

Consequently, the aim of this study was to examine whether routinely measured right-heart catheter (RHC) data correlate with 6MWT outcomes. Not only walk distance but also desaturation and dyspnea are included in outcomes and a comparison was made among these three.

\section{Methods}

2.1. Patients. Pulmonary function tests (PFTs), 6MWT, and $\mathrm{RHC}$ are part of the regular evaluation for patients with ILD when diagnosed at our university hospital. Inclusion criteria for this study were as follows: diagnosis of ILD; having undergone evaluation between June 2010 and December 2012; age $\geqq 18$ years; the absence of any recent (i.e., $<3$ months) acute worsening of ILD including infection, pulmonary embolism, and acute exacerbation of ILD; the absence of comorbid illness which might influence the evaluation of 6MWT and RHC, such as cardiovascular disease, chronic pulmonary embolism, COPD, and asthma. Patients with long-term oxygen therapy who could not remove their oxygen at the time of both 6MWT and RHC examinations were excluded. Patients with mean pulmonary artery pressure (PAP) $\geqq 35$ were also excluded because of possibility that pulmonary artery hypertension is the main pathophysiology in such patients. Since this study was based on retrospective analysis, the informed consent requirement was waived. The study protocol was approved by the ethics committee of Kindai University Faculty of Medicine (number 25-012).

2.2. PFTs. PFTs included spirometry and single-breath measurements of diffusing capacity (DLco) (CHESTAC-8800; Chest, Tokyo, Japan) and arterial blood gas analysis, which were all performed according to the American Thoracic Society standards $[17,18]$. Results were expressed in absolute values and as a percentage of Japanese normal predicted values [19].

2.3. Hemodynamic Studies. RHC was performed using a Swan-Ganz catheter percutaneously via either the cervical or femoral vein. Hemodynamic variables including mean PAP, systolic and diastolic right ventricular pressure, mean right arterial pressure (RAP), pulmonary capillary wedge pressure (PCWP), and mean systemic arterial pressure were measured. Cardiac output (CO) was also measured by the thermodilution method. Pulmonary vascular resistance was calculated by dividing (mean pulmonary artery pressure pulmonary capillary wedge pressure) by cardiac output.

2.4. 6MWT. 6MWT was conducted in all patients who participated in the study, according to the ATS statement [1]. Briefly, all patients were tested under the standardized condition by trained physicians. The patients wore lightweight pulse oximeters (WristOx ${ }^{\mathrm{TM}}$, NONIN, Inc., Minneapolis, USA), and baseline heart rate and transcutaneous oxygen saturation $\left(\mathrm{SpO}_{2}\right)$ were measured. Then, patients were instructed to walk as far as possible for $6 \mathrm{~min}$. The physician who conducted the test did not walk with the patients but checked and recorded $\mathrm{SpO}_{2}$ and heart rates on the pulse oximeter immediately after the test. The distance the patients walked and dyspnea rating immediately after the test were also recorded. To evaluate dyspnea rating, patients were asked to rate their dyspnea using the modified Borg scale, by selecting a number from 0 to 10 , with 0 being no appreciable dyspnea and 10 being maximal sustainable dyspnea [20].

2.5. Statistical Analyses. Pearson correlation coefficients $(r)$ between 6MWT outcomes and various physiological parameters including RHC data were determined. The 6MWT outcomes included walk distance, $\mathrm{SpO}_{2}$ at the end of $6 \mathrm{MWT}$, and dyspnea assessed with the Borg scale. The interrelationships among 6MWT outcomes were also examined. In the subsequent multivariate model, a stepwise regression analysis was performed using each 6MWT outcome as a dependent variable to select more significant variables. Any variables which had been revealed to have a significant correlation with the dependent variable were introduced in the multivariate model as potential predictors. As for pulmonary function variables such as forced vital capacity (FVC) and DLco, \% predicted values were used in the multivariate model to avoid the effect of anthropometric factors. All tests were performed at a significance level of 0.05 . Analyses were performed with the PASW statistical package ver.18 (SPSS Japan Inc., Tokyo, Japan), and all values are presented as mean \pm standard deviation (SD) unless otherwise indicated.

\section{Results}

3.1. Patient Characteristics. Forty-six consecutive patients with ILD were included in the study. Anthropometry and diagnoses of the patients are shown in Table 1. Of 46 patients, 20 were diagnosed with IPF according to the official ATS/ERS/JRS/ALAT statement [21]. Fourteen were diagnosed as having collagen vascular disease associated interstitial lung disease (CVD-ILD), which included 8 patients with rheumatoid arthritis, 3 with systemic sclerosis, 2 with dermatomyositis, and 2 with Sjögren's syndrome. The other 12 patients were not diagnosed with specific ILD, and therefore they were diagnosed as simply having idiopathic interstitial pneumonia (IIP). 
TABle 1: Patient characteristics.

\begin{tabular}{lc}
\hline Variables & Values \\
\hline Sex, number (\%) & \\
$\quad$ Male & $28(61)$ \\
Female & $18(39)$ \\
\hline Age, yr & $68.9 \pm 10.1$ \\
Height, cm & $159.2 \pm 8.8$ \\
Weight, kg & $58.1 \pm 12.6$ \\
\hline Diagnosis, number & \\
IPF & 20 \\
IIP (no specific diagnosis) & 12 \\
CVD-ILD & 14 \\
\hline
\end{tabular}

The number of patients was 46 . Data are presented as mean \pm standard deviation (SD), unless otherwise indicated.

IPF: idiopathic pulmonary fibrosis; IIP: idiopathic interstitial pneumonia; CVD-ILD: connective tissue disease associated interstitial lung disease.

3.2. PFT, Arterial Gas Analysis, Hemodynamics, and 6MWT Outcomes. PFT data, arterial blood analysis, hemodynamics, and results of 6MWT are shown in Table 2. The patients had a mild decrease in \% predicted FVC, consistent with a mild restrictive defect. Reduction in DLco was moderate and decrease in $\mathrm{PaO}_{2}$ was relatively mild. RHC data show the mean PAP and mean PCWP within a normal range. However, there were 2 patients whose mean PAP was $\geqq 25 \mathrm{mmHg}$, high enough to be diagnosed with pulmonary hypertension [22, 23]. The mean PAPs of each of these 2 patients were 26 with IIP and 27 with dermatomyositis. In 6MWT outcomes, the mean walk distance was $394 \mathrm{~m}$, although the distance was widely distributed from person to person. The mean end-test $\mathrm{SpO}_{2}$ was $85.4 \%$, showing mild desaturation. In fact, 23 of 46 patients $(50.0 \%)$ showed desaturation of less than $88 \%$ at the end of the 6MWT. The mean dyspnea level assessed with the modified Borg scale was 3.7, although it ranged between extremes. As shown in Figure 1, actual values of each outcome were widely distributed.

3.3. Correlates of $6 M W T$ Outcomes. The results of single correlation analysis for 6MWT outcomes are shown in Table 3. For walk distance, weight $(r=0.31, p=0.04)$, FVC $(r=0.49, p=0.0005), \%$ predicted FVC $(r=0.39$, $p=0.007)$, DLco $(r=0.61, p<0.0001), \%$ predicted DLco $(r=0.46, p=0.002), \mathrm{PaO}_{2}(r=0.43, p=0.002)$, and PVR $(r=-0.33, p=0.02)$ were revealed to be significant correlates.

For $\mathrm{SpO}_{2}$ at the end of 6MWT, DLco $(r=0.47, p=$ $0.001), \%$ predicted DLco $(r=0.49, p=0.0007), \mathrm{PaO}_{2}$ $(r=0.43, p=0.003)$, mean PAP $(r=-0.50, p=0.0003)$, and PVR $(r=-0.63, p<0.0001)$ were significant correlates.

For dyspnea at end of 6MWT assessed with the modified Borg scale, factors which showed significant correlation were DLco $(r=-0.38, p=0.01)$, \% predicted DLco $(r=-0.42$, $p=0.004)$, and mean PAP $(r=0.32, p=0.03)$.

The results of stepwise multivariate regression analyses which were performed to reveal more significant parameters are shown in Table $4 . \%$ predicted FVC and \% predicted DLco were as independent predictors of walk distance $\left(r^{2}=0.35\right.$,
TABLE 2: Pulmonary function, arterial gas analysis, hemodynamics, and 6 min walk test outcomes.

\begin{tabular}{|c|c|c|}
\hline Variables & Mean \pm SD & Range \\
\hline \multicolumn{3}{|l|}{ Pulmonary function } \\
\hline FVC, L & $2.3 \pm 0.8$ & $0.9-4.4$ \\
\hline FVC, \% predicted & $76.7 \pm 17.1$ & $41.5-122.0$ \\
\hline $\mathrm{DLco}, \mathrm{mL} / \mathrm{min} / \mathrm{mmHg}$ & $10.1 \pm 3.2$ & $4.8-17.0$ \\
\hline DLco, $\%$ predicted & $62.9 \pm 16.0$ & $37.8-118.1$ \\
\hline \multicolumn{3}{|l|}{ Arterial gas analysis } \\
\hline $\mathrm{PaO}_{2}, \mathrm{mmHg}$ & $76.6 \pm 10.6$ & $58.0-101.7$ \\
\hline $\mathrm{PaCO}_{2}, \mathrm{mmHg}$ & $40.6 \pm 4.4$ & $31.1-48.8$ \\
\hline $\mathrm{pH}$ & $7.41 \pm 0.02$ & $7.38-7.47$ \\
\hline \multicolumn{3}{|l|}{ Hemodynamics } \\
\hline Mean AP, mmHg & $91.3 \pm 12.3$ & $66.6-114.0$ \\
\hline RAP, $\mathrm{mmHg}$ & $2.9 \pm 2.8$ & $0.4-8.1$ \\
\hline Mean PAP, mmHg & $16.6 \pm 4.8$ & $7.0-27.0$ \\
\hline PCWP, mmHg & $6.5 \pm 3.3$ & $0-15.0$ \\
\hline $\mathrm{CO}, \mathrm{L} / \mathrm{min}$ & $4.7 \pm 1.1$ & $3.0-8.0$ \\
\hline Cardiac index, $\mathrm{L} / \mathrm{min} / \mathrm{m}^{2}$ & $3.0 \pm 0.6$ & $1.8-4.6$ \\
\hline PVR, dyne.s $/ \mathrm{cm}^{5}$ & $177 \pm 81$ & $0-342$ \\
\hline \multicolumn{3}{|l|}{ 6MWT outcomes } \\
\hline Walk distance, $\mathrm{m}$ & $394 \pm 102$ & $120-580$ \\
\hline $\mathrm{SpO}_{2}$ at end of the test, $\%$ & $85.4 \pm 8.3$ & $62-97$ \\
\hline Dyspnea at end of the test & $3.7 \pm 2.6$ & $0-10$ \\
\hline
\end{tabular}

The number of patients was 46 except for DLco which was 44 because 2 could not perform the examination for $\%$ DLco due to low pulmonary function.

Data are presented as mean \pm standard deviation (SD). Dyspnea was assessed with the modified Borg scale.

FVC: forced vital capacity; DLco: diffusing capacity of carbon monoxide; $\mathrm{PaO}_{2}$ : partial arterial pressure of oxygen; $\mathrm{PaCO}_{2}$ : partial arterial pressure of carbon dioxide; AP: systemic arterial pressure; RAP: right arterial pressure; PAP: pulmonary artery pressure; PCWP: pulmonary capillary wedge pressure; CO: cardiac output; PVR: pulmonary vascular resistance; $\mathrm{SpO}_{2}$ : transcutaneous oxygen saturation; 6MWT: 6 min walk test.

$p=0.0002$ ). For $\mathrm{SpO}_{2}$ at the end of $6 \mathrm{MWT}, \%$ predicted DLco and PVR were selected as independent predictors $\left(r^{2}=\right.$ $0.46, p<0.0001)$. For dyspnea at the end of $6 \mathrm{MWT}$, only $\%$ predicted DLco was a significant independent predictor $\left(r^{2}=0.18, p=0.005\right)$.

\section{Discussion}

In the present study, we analyzed whether pulmonary hemodynamics significantly correlate with and predict each $6 \mathrm{MWT}$ outcome, including walk distance, $\mathrm{SpO}_{2}$, and dyspnea at the end of the test, and also examined to see if there were any differences among different 6MWT outcomes in patients with ILD who were nonselectively recruited. As results of univariate analysis, mean PAP and/or PVR as well as $\%$ predicted DLco were significantly correlated with all 3 outcomes of $6 \mathrm{MWT}$. In stepwise multivariate regression analyses, pulmonary hemodynamics data was excluded from significant correlates of 6MWT outcomes except $\mathrm{SpO}_{2}$ at end of 6MWT. Furthermore, some differences were observed among predictors for 3 outcomes of $6 \mathrm{MWT}$. As for walk 

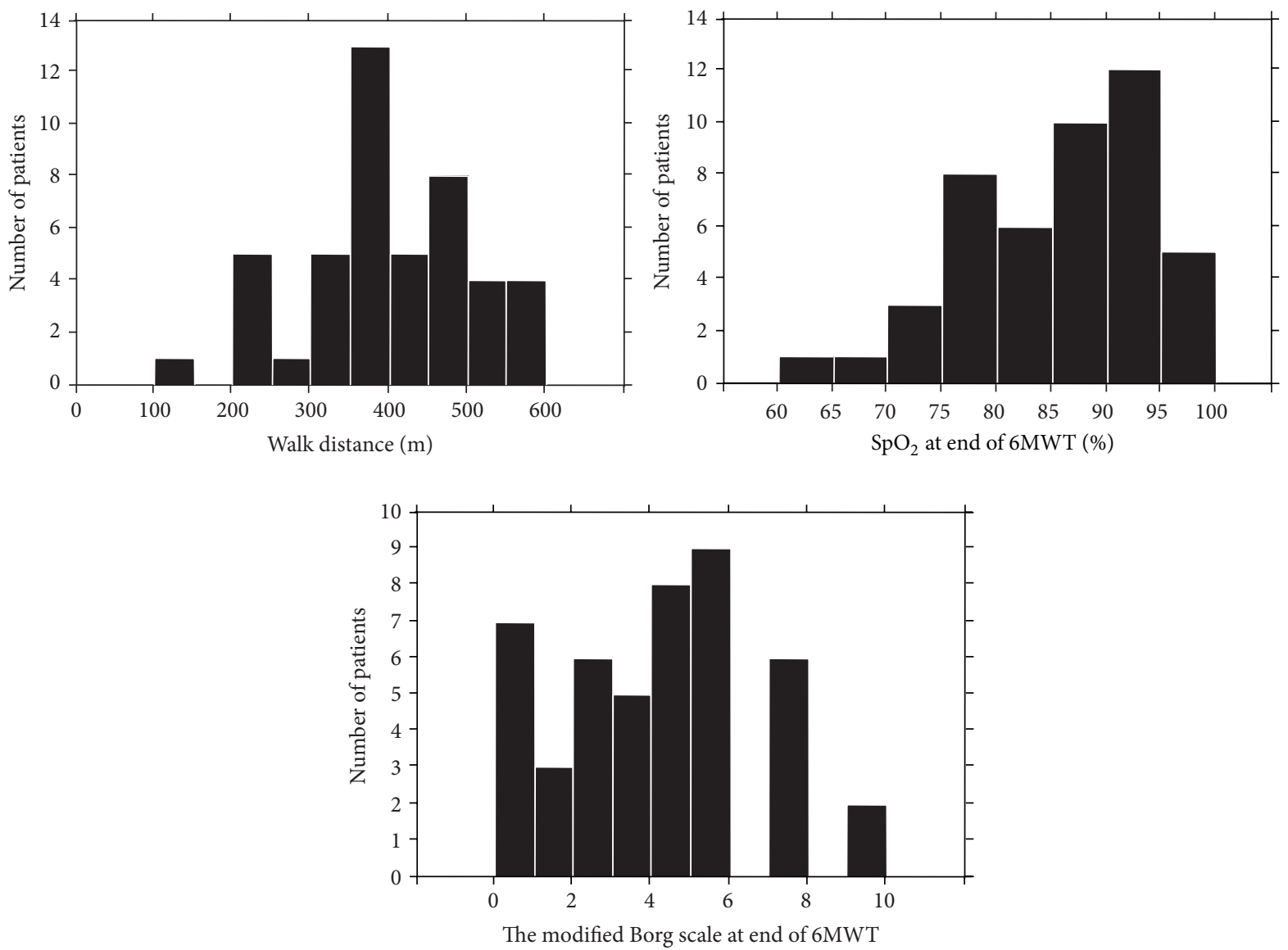

Figure 1: Histogram of patients for 6-min walk distance, $\mathrm{SpO}_{2}$ at end of $6 \mathrm{MWT}$, and dyspnea assessed with modified Borg scale at the end of the test. $\mathrm{SpO}_{2}$ transcutaneous oxygen saturation; 6MWT: 6-min walk test.

distance, \% predicted FVC and \% predicted DLco were independent predictors. In contrast, as for $\mathrm{SpO}_{2}$ at the end of the test, \% predicted DLco and PVR were independent predictors. As for dyspnea at the end of the test, \% predicted DLco was only one predictor.

In patients with COPD, several factors which predict 6min walk distance have been reported. It has been demonstrated that 6-min walk distance is shorter in patients with an advanced age and a higher BMI, in patients with severe airflow obstruction, and in women $[1,4-6]$. However, these factors explain only a small part of the variation among individuals [24]. Some other factors which increase exertional dyspnea, such as dynamic hyperinflation, may play a role in the variability of walk distance [24].

There have been some reports examining the impact of pulmonary hemodynamics on exercise capacity in ILD [2528]. Those reports equally demonstrated that mPAP had significant negative impact on exercise capacity. In our study, however, mPAP was not selected as predictor of any $6 \mathrm{MWT}$ outcomes in multivariate analysis, although it was correlated with each outcome of $6 \mathrm{MWT}$ in univariate analysis. It seems that this discrepancy arose because of differences in patient characteristics. Only $4 \%$ of patients had pulmonary hypertension of $\geqq 25 \mathrm{mmHg}$ in our study while more patients did in other studies. One of the reasons for this difference may be that RHC was performed as part of a routine regular evaluation for ILD in our hospital. Another reason may be that some patients with pulmonary hypertension could have been be excluded from our study, because patients with longterm oxygen therapy who could not discontinued their oxygen apparatus at the time of 6MWT and RHC examinations both were excluded from the study. The exclusion criteria for the present study of patients with mean PAP $\geqq 35$ also might contribute. For these reasons, it can be said that the results of the present study reflect characteristics of patients with mean PAP within normal range to mild pulmonary hypertension, which also may reflect the real world in clinical practice. The presence of pulmonary hypertension is important and is one of predicting factors of longevity in IPF patients [13-16]. However, in patient populations with mean PAP within normal range to mild pulmonary hypertension, pulmonary artery pressure had little influence on 6MWT outcomes in multivariate analysis, although even pulmonary artery pressure less than $25 \mathrm{mmHg}$ reportedly influences the longevity of patients with IPF [15]. It can be assumed in clinical practice that the presence of pulmonary hypertension should be investigated separately from evaluations for pulmonary function and exercise capacity in ILD patients. Of 
TABLE 3: Pearson correlation coefficients between 6 min walk test outcomes and various parameters.

\begin{tabular}{lcccccc}
\hline Variables & \multicolumn{2}{c}{ Walk } & \multicolumn{2}{c}{$\begin{array}{c}\mathrm{SpO}_{2} \text { at the } \\
\text { end of } 6 \mathrm{MWT}\end{array}$} & \multicolumn{2}{c}{$\begin{array}{c}\text { Borg scale at } \\
\text { the end of }\end{array}$} \\
& $r$ & $p$ & $r$ & $p$ & $r$ & $p$ TW \\
\hline Age & -0.20 & 0.19 & -0.27 & 0.07 & 0.06 & 0.68 \\
Height & 0.23 & 0.12 & 0.16 & 0.29 & -0.10 & 0.51 \\
Weight & 0.31 & 0.04 & -0.10 & 0.51 & -0.03 & 0.84 \\
BMI & 0.26 & 0.08 & -0.27 & 0.07 & 0.05 & 0.76 \\
FVC & 0.49 & 0.0005 & 0.12 & 0.44 & -0.23 & 0.12 \\
\% FVC & 0.39 & 0.007 & -0.04 & 0.80 & -0.22 & 0.14 \\
DLco & 0.61 & $<0.0001$ & 0.47 & 0.001 & -0.38 & 0.01 \\
\% DLco & 0.46 & 0.002 & 0.49 & 0.0007 & -0.42 & 0.004 \\
PaO & 0.43 & 0.002 & 0.43 & 0.003 & -0.30 & 0.04 \\
PaCO & 0.17 & 0.27 & -0.09 & 0.56 & -0.12 & 0.47 \\
Mean AP & -0.01 & 0.95 & 0.02 & 0.90 & 0.08 & 0.60 \\
RAP & 0.10 & 0.52 & 0.26 & 0.09 & -0.04 & 0.79 \\
Mean PAP & -0.27 & 0.07 & -0.50 & 0.0003 & 0.32 & 0.03 \\
PCWP & 0.004 & 0.98 & 0.01 & 0.94 & -0.02 & 0.90 \\
CO $_{\text {Cardiac }}$ & -0.05 & 0.76 & 0.16 & 0.29 & 0.14 & 0.36 \\
index & -0.13 & 0.65 & 0.10 & 0.52 & 0.17 & 0.26 \\
PVR & -0.33 & 0.02 & -0.63 & $<0.0001$ & 0.24 & 0.12 \\
\hline
\end{tabular}

The number of patients was 46 except for DLco, which numbered 44 . BMI: body mass index; FVC: forced vital capacity; \% FVC: \% predicted FVC; DLco: diffusing capacity of carbon monoxide; \% DLco: \% predicted DLco; $\mathrm{PaO}_{2}$ : partial arterial pressure of oxygen; $\mathrm{PaCO}_{2}$ : partial arterial pressure of carbon dioxide; AP: systemic arterial pressure; RAP: right arterial pressure; PAP: pulmonary artery pressure; PCWP: pulmonary capillary wedge pressure; CO: cardiac output; PVR: pulmonary vascular resistance; $\mathrm{SpO}_{2}$ : transcutaneous oxygen saturation; 6MWT: 6 min walk test.

course, further study is needed to confirm the importance of pulmonary artery pressure on 6MWT outcomes in each specific disease.

However, a PVR was selected as one of the predictors of desaturation at 6MWT, although it was not correlated with 6min walk distance. Abnormality in pulmonary hemodynamic influences only desaturation after exercise in this patient population.

The present study should be considered in light of its strengths and limitations. Analysis including hemodynamic variables measured with routine RHC examination allowed us to exclude selection bias. If RHC was performed only for patients who most likely had pulmonary hypertension, the rate of patients with severe lung impairment or with a factor of pulmonary artery hypertension would have increased, and the patient population would have been slanted. On the contrary, there are some limitations which should be addressed in the present study. First, as mentioned above, it was possible that some patients with pulmonary hypertension were excluded from the study, because patients who could not take off their oxygen apparatus at the time of both examinations were excluded. If more patients with severe pulmonary hypertension $\geqq 25 \mathrm{mgHg}$ of mPAP were included
TABLE 4: Results of stepwise multiple regression analysis for walk distance and $\mathrm{SpO}_{2}$ and the Borg scale at end of 6 min walk test.

\begin{tabular}{lcc}
\hline Variables & $r$ & $p$ \\
\hline For walk distance & - & - \\
Weight & 0.37 & 0.01 \\
$\% \mathrm{FVC}$ & 0.42 & 0.003 \\
$\% \mathrm{DLco}$ & - & - \\
$\mathrm{PaO}_{2}$ & - & - \\
$\mathrm{PVR}^{\text {For SpO}}$ at end of 6MWT & & \\
$\% \mathrm{DLco}^{2}$ & 0.31 & 0.001 \\
$\mathrm{PaO}$ & - & - \\
$\mathrm{Mean}_{2}$ PAP & - & - \\
$\mathrm{PVR}$ & -0.51 & $<0.0001$ \\
\hline For the Borg scale at end of 6MWT & & \\
$\%$ DLco & -0.42 & 0.004 \\
Mean PAP & - & - \\
\hline
\end{tabular}

The number of patients was 44 because 2 were excluded from the analysis due to absence of \% DLco. $r$ is presented as a partial correlation coefficient. $r$ is presented only for variables which were selected as significant by the stepwise multiple regression analysis. Cumulative $r^{2}$ was $0.35(p=0.0002)$ for walk distance, $0.46(p<0.0001)$ for $\mathrm{SpO}_{2}$ at end of $6 \mathrm{MWT}$, and $0.18(p=0.005)$ for the Borg scale at end of 6MWT, respectively.

$\%$ FVC: predicted forced vital capacity; \% DLco: \% predicted diffusing capacity of carbon monoxide; $\mathrm{PaO}_{2}$ : partial arterial pressure of oxygen; PAP: pulmonary artery pressure; $\mathrm{PVR}$ : pulmonary vascular resistance; $\mathrm{SpO}_{2}$ : transcutaneous oxygen saturation; 6MWT: 6 min walk test.

in the study, other results might have been obtained. It may also be true that ILD patients with pulmonary hypertension should be investigated separately. Second, the diagnosis of diseases varied. The patient population consisted of IPF, IIP with no specific classification, and CVD-ILD. Because differences might exist among pathophysiologies of ILD, further study which enrolls patients with a specific disorder, such as IPF only, should be considered.

In conclusion, \% predicted FVC and \% predicted DLco were predictors of 6-min walk distance. \% predicted DLco and PVR were predictors of desaturation at 6MWT. Only $\%$ predicted DLco was a predictor of dyspnea at 6MWT in patients with ILD. Consequently, mean PAP had little impact on 6MWT outcomes in ILD patients who were nonselectively recruited, although a PVR was one of predictors of desaturation.

\section{Competing Interests}

The authors declare they have no related conflict of interests.

\section{Authors' Contributions}

Osamu Nishiyama and Ryo Yamazaki collected data, Osamu Nishiyama did statistical analysis and wrote the paper, Hiroyuki Sano, Takashi Iwanaga, Yuji Higashimoto, and Hiroaki Kume reviewed the paper and made revisions, and Yuji Tohda made final version of the paper. 


\section{References}

[1] ATS Committee on Proficiency Standards for Clinical Pulmonary Function Laboratories, "ATS statement: guidelines for the six-minute walk test," American Journal of Respiratory and Critical Care Medicine, vol. 166, pp. 111-117, 2002.

[2] P. Palange, S. A. Ward, K.-H. Carlsen et al., "Recommendations on the use of exercise testing in clinical practice," European Respiratory Journal, vol. 29, no. 1, pp. 185-209, 2007.

[3] R. Arena and K. E. Sietsema, "Cardiopulmonary exercise testing in the clinical evaluation of patients with heart and lung disease," Circulation, vol. 123, no. 6, pp. 668-680, 2011.

[4] P. L. Enright, M. A. McBurnie, V. Bittner et al., "The 6-min walk test: a quick measure of functional status in elderly adults," Chest, vol. 123, no. 2, pp. 387-398, 2003.

[5] P. L. Enright, “The six-minute walk test," Respiratory Care, vol. 48, no. 8, pp. 783-785, 2003.

[6] E. Ischaki, G. Papatheodorou, E. Gaki, I. Papa, N. Koulouris, and S. Loukides, "Body mass and fat-free mass indices in COPD: relation with variables expressing disease severity," Chest, vol. 132, no. 1, pp. 164-169, 2007.

[7] V. N. Lama, K. R. Flaherty, G. B. Toews et al., "Prognostic value of desaturation during a 6 minute walk test in idiopathic interstitial pneumonia," American Journal of Respiratory and Critical Care Medicine, vol. 168, no. 9, pp. 1084-1090, 2003.

[8] T. S. Hallstrand, L. J. Boitano, W. C. Johnson, C. A. Spada, J. G. Hayes, and G. Raghu, "The timed walk test as a measure of severity and survival in idiopathic pulmonary fibrosis," European Respiratory Journal, vol. 25, no. 1, pp. 96-103, 2005.

[9] D. J. Lederer, S. M. Arcasoy, J. S. Wilt, F. D’Ovidio, J. R. Sonett, and S. M. Kawut, "Six-minute-walk distance predicts waiting list survival in idiopathic pulmonary fibrosis," American Journal of Respiratory and Critical Care Medicine, vol. 174, no. 6, pp. 659-664, 2006.

[10] K. R. Flaherty, A.-C. Andrei, S. Murray et al., "Idiopathic pulmonary fibrosis: prognostic value of changes in physiology and six-minute-walk test," American Journal of Respiratory and Critical Care Medicine, vol. 174, no. 7, pp. 803-809, 2006.

[11] O. Nishiyama, H. Taniguchi, Y. Kondoh et al., "A simple assessment of dyspnoea as a prognostic indicator in idiopathic pulmonary fibrosis," European Respiratory Journal, vol. 36, no. 5, pp. 1067-1072, 2010.

[12] O. Nishiyama, H. Taniguchi, Y. Kondoh et al., "Dyspnoea at 6-min walk test in idiopathic pulmonary fibrosis: comparison with COPD," Respiratory Medicine, vol. 101, no. 4, pp. 833-838, 2007.

[13] H. F. Nadrous, P. A. Pellikka, M. J. Krowka et al., "Pulmonary hypertension in patients with idiopathic pulmonary fibrosis," Chest, vol. 128, no. 4, pp. 2393-2399, 2005.

[14] C. J. Lettieri, S. D. Nathan, S. D. Barnett, S. Ahmad, and A. F. Shorr, "Prevalence and outcomes of pulmonary arterial hypertension in advanced idiopathic pulmonary fibrosis," Chest, vol. 129, no. 3, pp. 746-752, 2006.

[15] K. Hamada, S. Nagai, S. Tanaka et al., "Significance of pulmonary arterial pressure and diffusion capacity of the lung as prognosticator in patients with idiopathic pulmonary fibrosis," Chest, vol. 131, no. 3, pp. 650-656, 2007.

[16] M. Kimura, H. Taniguchi, Y. Kondoh et al., "Pulmonary hypertension as a prognostic indicator at the initial evaluation in idiopathic pulmonary fibrosis," Respiration, vol. 85, no. 6, pp. 456-463, 2013.
[17] "Standardization of Spirometry, 1994 Update. American Thoracic Society," American Journal of Respiratory and Critical Care Medicine, vol. 152, no. 3, pp. 1107-1136, 1995.

[18] American Thoracic Society, "Single-breath carbon monoxide diffusing capacity (transfer factor). Recommendations for a standard technique-1995 update," American Journal of Respiratory and Critical Care Medicine, vol. 152, no. 6, pp. 2185-2198, 1995.

[19] S. Hanamoto, T. Ohsuji, I. Tsuyuguchi, S. Kawabata, and K. Kimura, "Prediction formulas for pulmonary function tests expressed in linear and exponential form for healthy Japanese adults," Nihon Kyobu Shikkan Gakkai Zasshi, vol. 30, no. 12, pp. 2051-2060, 1992.

[20] G. A. V. Borg, "Psychophysical bases of perceived exertion," Medicine and Science in Sports and Exercise, vol. 14, no. 5, pp. 377-381, 1982.

[21] G. Raghu, H. R. Collard, J. J. Egan et al., "An official ATS/ERS/JRS/ALAT statement: idiopathic pulmonary fibrosis: evidence-based guidelines for diagnosis and management," American Journal of Respiratory and Critical Care Medicine, vol. 183, no. 6, pp. 788-824, 2011.

[22] N. Galie, M. M. Hoeper, M. Humbert et al., "Guidelines for the diagnosis and treatment of pulmonary hypertension: the Task Force for the Diagnosis and Treatment of Pulmonary Hypertension of the European Society of Cardiology (ESC) and the European Respiratory Society (ERS), endorsed by the International Society of Heart and Lung Transplantation (ISHLT)," European Heart Journal, vol. 30, pp. 2493-2537, 2009.

[23] G. Simonneau, I. M. Robbins, M. Beghetti et al., "Updated clinical classification of pulmonary hypertension," Journal of the American College of Cardiology, vol. 54, no. 1, pp. S43-S54, 2009.

[24] J. M. Marin, S. J. Carrizo, M. Gascon, A. Sanchez, B. Gallego, and B. R. Celli, "Inspiratory capacity, dynamic hyperinflation, breathlessness, and exercise performance during the 6-minutewalk test in chronic obstructive pulmonary disease," American Journal of Respiratory and Critical Care Medicine, vol. 163, no. 6, pp. 1395-1399, 2001.

[25] O. A. Minai, J. F. Santacruz, J. M. Alster, M. M. Budev, and K. McCarthy, "Impact of pulmonary hemodynamics on 6min walk test in idiopathic pulmonary fibrosis," Respiratory Medicine, vol. 106, no. 11, pp. 1613-1621, 2012.

[26] S. Gläser, O. Noga, B. Koch et al., "Impact of pulmonary hypertension on gas exchange and exercise capacity in patients with pulmonary fibrosis," Respiratory Medicine, vol. 103, no. 2, pp. 317-324, 2009.

[27] A. K. Boutou, G. G. Pitsiou, I. Trigonis et al., "Exercise capacity in idiopathic pulmonary fibrosis: the effect of pulmonary hypertension," Respirology, vol. 16, no. 3, pp. 451-458, 2011.

[28] H. F. Armstrong, P. C. Schulze, M. Bacchetta, W. Thirapatarapong, and M. N. Bartels, "Impact of pulmonary hypertension on exercise performance in patients with interstitial lung disease undergoing evaluation for lung transplantation," Respirology, vol. 19, no. 5, pp. 675-682, 2014. 


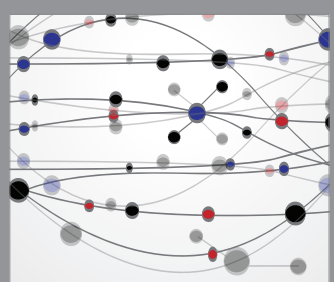

The Scientific World Journal
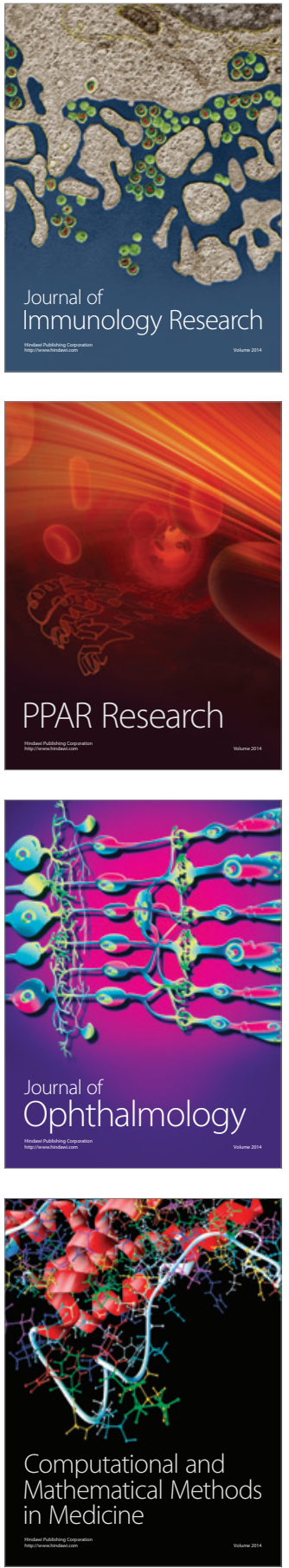

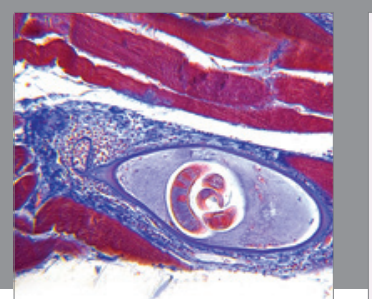

Gastroenterology Research and Practice

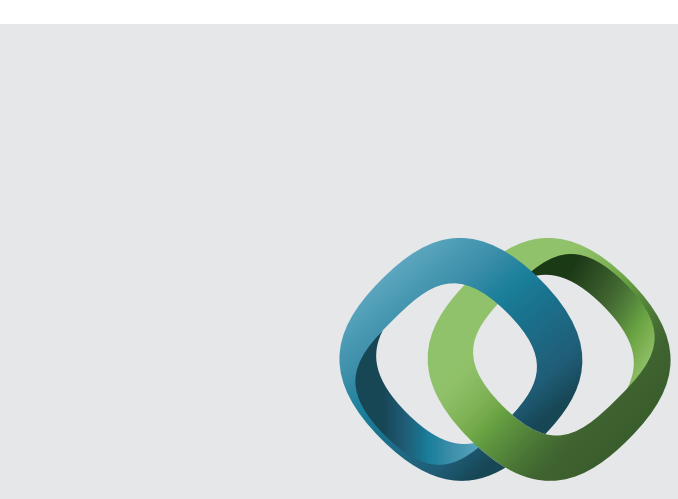

\section{Hindawi}

Submit your manuscripts at

http://www.hindawi.com
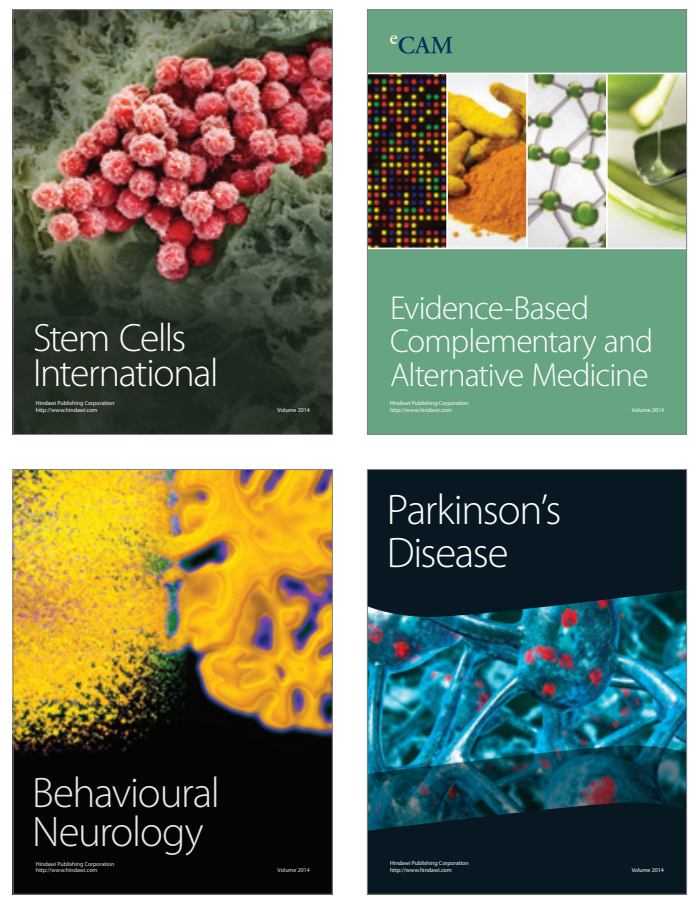
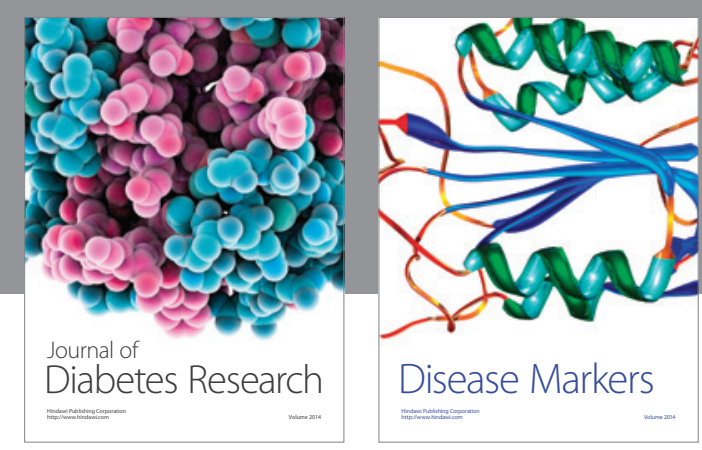

Disease Markers
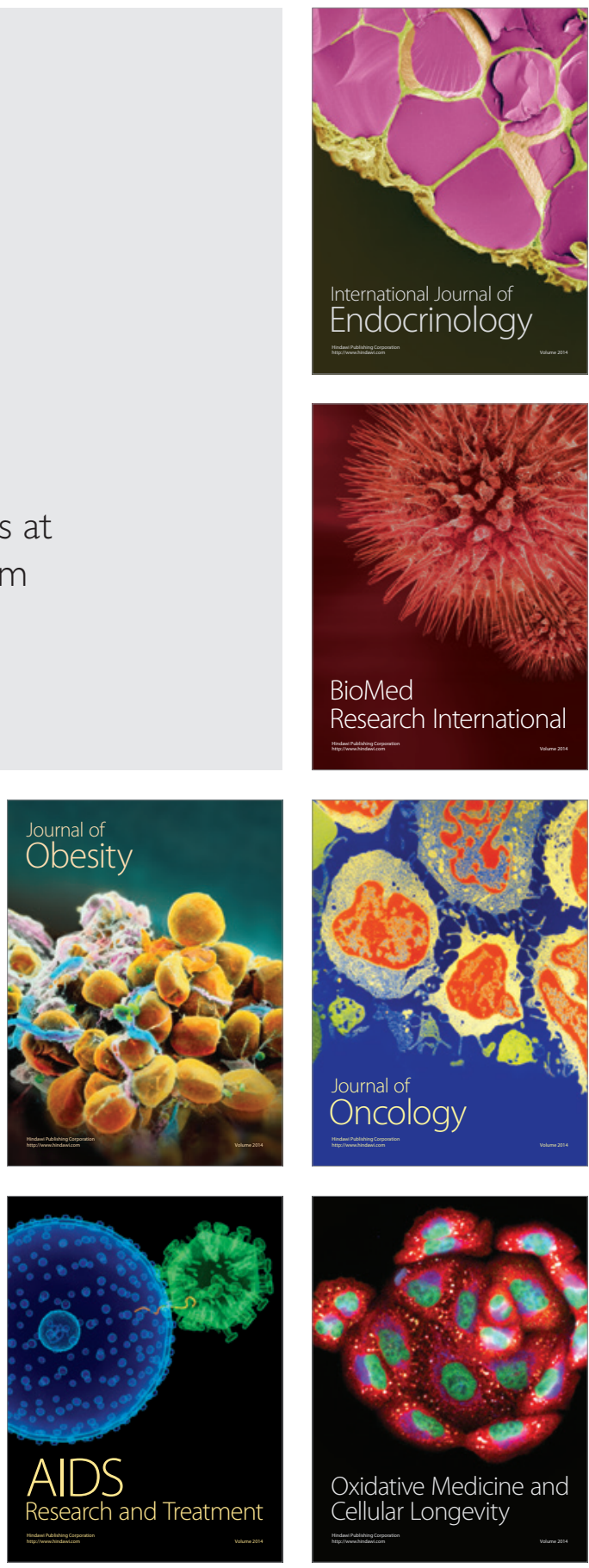